\title{
Increased COVID-19 Infection Risk Drives Racial and Ethnic Disparities in Severe COVID-19 Outcomes
}

\author{
Susan M. Shortreed ${ }^{1,2}$ (D) Regan Gray ${ }^{1} \cdot$ Mary Abisola Akosile ${ }^{1} \cdot$ Rod L. Walker $^{1} \cdot$ Sharon Fuller $^{1} \cdot$ Lisa Temposky $^{1}$. \\ Stephen P. Fortmann ${ }^{3}$. Ladia Albertson-Junkans ${ }^{1}$. James S. Floyd ${ }^{4,5}$. Elizabeth A. Bayliss ${ }^{6,7}$. Laura B. Harrington ${ }^{1,5}$. \\ Mi H. Lee ${ }^{3}$. Sascha Dublin ${ }^{1,5}$
}

Received: 12 October 2021 / Revised: 2 December 2021 / Accepted: 3 December 2021 / Published online: 24 January 2022

c) W. Montague Cobb-NMA Health Institute 2022

\begin{abstract}
COVID-19 inequities have been well-documented. We evaluated whether higher rates of severe COVID-19 in racial and ethnic minority groups were driven by higher infection rates by evaluating if disparities remained when analyses were restricted to people with infection. We conducted a retrospective cohort study of adults insured through Kaiser Permanente (Colorado, Northwest, Washington), follow-up in March-September 2020. Laboratory results and hospitalization diagnosis codes identified individuals with COVID-19. Severe COVID-19 was defined as invasive mechanical ventilation or mortality. Self-reported race and ethnicity, demographics, and medical comorbidities were extracted from health records. Modified Poisson regression estimated adjusted relative risks (aRRs) of severe COVID-19 in full cohort and among individuals with infection. Our cohort included 1,052,774 individuals, representing diverse racial and ethnic minority groups (e.g., 68,887 Asian, 41,243 Black/African American, 93,580 Hispanic or Latino/a individuals). Among 7,399 infections, 442 individuals experienced severe COVID-19. In the full cohort, severe COVID-19 aRRs for Asian, Black/African American, and Hispanic individuals were 2.09 (95\% CI: 1.36, 3.21), 2.02 (1.39, 2.93), and 2.09 (1.57, 2.78), respectively, compared to non-Hispanic Whites. In analyses restricted to individuals with COVID-19, all aRRs were near 1, except among Asian Americans (aRR $1.82[1.23,2.68])$. These results indicate increased incidence of severe COVID-19 among Black/African American and Hispanic individuals is due to higher infection rates, not increased susceptibility to progression. COVID-19 disparities most likely result from social, not biological, factors. Future work should explore reasons for increased severe COVID-19 risk among Asian Americans. Our findings highlight the importance of equity in vaccine distribution.
\end{abstract}

Keywords Health equity $\cdot$ Health impacts of structural racism $\cdot$ COVID-19 disease severity $\cdot$ Infection $\cdot$ Health disparities

Susan M. Shortreed

susan.m.shortreed@kp.org

1 Kaiser Permanente Washington Health Research Institute, 1730 Minor Avenue, Ste 1600, Seattle, WA 98101, USA

2 Department of Biostatistics, University of Washington, F-600, Health Sciences Building, 1705 NE Pacific Street, Seattle, WA 98195-7232, USA

3 Kaiser Permanente Center for Health Research, 3800 N. Interstate Ave, Portland, OR 97227, USA

4 Department of Medicine, University of Washington, RR-512, Health Sciences Building, 1959 NE Pacific Street, Seattle, WA 98195, USA
5 Department of Epidemiology, University of Washington, 3980 15th Ave NE, Seattle, WA 98195, USA

6 Institute for Health Research, Kaiser Permanente Colorado, 2550 S. Parker Rd, Suite 200, Aurora, CO 80014, USA

7 Department of Family Medicine, University of Colorado School of Medicine, 12631 East 17th Ave, Box F 496, Aurora, CO 80045, USA 


\section{Introduction}

The disparities across racial and ethnic groups in the impact of COVID-19 in the United States (US) are wellestablished. Severe COVID-19, including death, has affected Asian American, Black and African American (BA/AA), Hispanic and Latina/Latino (HI/LA), and Indigenous American and Alaskan Native (IA/AN) communities at disproportionately high rates [1-5]. Articles have pointed to the cause of this increased risk: structural racism [1-3, 6-8], which has broad implications for individuals' health and the quality of health care received [8-10], where and how individuals live[11], and the amount and type of their employment $[2,8]$. Racial discrimination within healthcare systems and its impact on care delivery have been shown to contribute to differences in health outcomes [12-14]. Racial and ethnic minority populations are more likely to have jobs classified as essential while having less access to jobs with health insurance, flexible work from home schedules, and paid leave while sick or awaiting COVID-19 test results [8]. Housing disparities have led to unequal access to COVID-19 testing and differences in the ability to quarantine (e.g., apartment building versus single-family home) $[7,9,11,15,16]$.

Our goal was to investigate if racial and ethnic disparities in severe COVID-19 were observed within a defined population of US adults with health insurance, and whether these disparities were primarily explained by increased risk of acquiring COVID-19 infection or progressing once infected (or both). In this cohort, information from severe acute respiratory syndrome coronavirus 2 (SARS-CoV-2) laboratory tests, hospitalization diagnosis codes, and death records allowed for assessment of primary COVID-19 infection and progression to severe disease, defined as invasive mechanical ventilation or death. If the risk of severe COVID-19 was different across racial and ethnic groups in the full population, but the same in the subset of individuals with COVID-19 infection, it would imply that observed disparities in severe COVID19 were driven by increased infection rates among racial and ethnic minority groups.

\section{Methods}

\section{Setting, Population, and Data Sources}

We studied adult (18 years and older) individuals insured by and receiving care in the Colorado (Colorado State), Northwest (Oregon State, Southwest Washington State), and Washington (Washington State) regions of Kaiser
Permanente (KP), a US health system that provides health care and health insurance. As of the cohort index date, February 29, 2020, individuals had to be continuously enrolled in their health system since September 1, 2019, or earlier (i.e., at least 6 months prior enrollment in their health system). Individuals with a COVID-19 infection prior to March 1, 2020, were excluded. Data were gathered from electronic health records (EHRs), insurance billing and other health system administrative sources, and state mortality certificates. Appropriate Institutional Review Boards approved this study.

\section{Outcomes}

COVID-19 infections were identified between March 1 and September 30, 2020. Infection was defined as present based on (1) EHR-recorded positive polymerase chain reaction (PCR) or nucleic acid amplification (NAA) tests; (2) hospital admissions with a COVID-19 International Classification Diseases version 10 (ICD-10) diagnosis code ("B342," “B9721," "B9729," "U071," "U072”), or (3) state death certificate with cause of death (COD) listed as COVID-19 or SARS infection due to coronavirus ("B342," "B9721," "B9729," "U071," “U072"). Severe COVID-19 was defined as hospitalization with invasive mechanical ventilation (see Appendix for codes used) or COVID-19-related death. COVID-19-related death was defined as a COVID-19 hospitalization with discharge status of expired, state death certificate COD listed as COVID-19 or SARS infection, or death within 28 days of COVID-19 infection. Interim (i.e., not finalized) state mortality certificates with cause of death (COD) information were available at KP Colorado and Washington through September 30, 2020; COD information was not available for KP Northwest. A sample of medical records from all three healthcare systems was reviewed to validate COVID-19 infection and invasive mechanical ventilation identified from diagnosis codes; positive predictive value for confirmed COVID-19 infection among hospitalizations with COVID-19 diagnosis codes was 96\% (73/76; 95\% confidence interval [CI] 89-99\%) and for invasive mechanical ventilation identified through diagnosis and procedures codes was $100 \%(25 / 25 ; 95 \%$ CI $86-100 \%)$.

\section{Race and Ethnicity}

Self-reported race and ethnicity was gathered from EHRs. Individuals provide race and ethnicity information at clinic visits; in some clinics, HI/LA ethnicity was gathered through a separate ethnicity question, while in other clinics, ethnicity was included as a race category. We grouped individuals using both race and ethnicity information; non-HI/LA White individuals, the most privileged group in the US, was the reference group in all analyses. People who self-identified 
as HI/LA were classified as such, while all other individuals were grouped using all race information provided. In this study, individuals were considered multiracial if they did not identify as HI/LA and identified with at least two races other than White; individuals who selected two race categories, one of which was White, were categorized into the selected racial group that was not White.

\section{Covariates}

Covariates were calculated as of February 29, 2020 (cohort entry). Age, gender, body mass index (BMI; $<25,25-30$, $30+\mathrm{kg} / \mathrm{m}^{2}$ ), and smoking status (current, former, never) were extracted from the EHR; individuals with no smoking information recorded were classified as never smokers. Hypertension, asthma, atherosclerotic cardiovascular disease (cerebrovascular disease, myocardial infarction, peripheral vascular disease), chronic obstructive pulmonary disease (COPD), and heart failure (HF) were identified through ICD-10 codes in the year prior. Diabetes mellitus (DM) and chronic kidney disease (CKD) were identified using both ICD-10 codes and lab results, and DM was also identified through use of DM medication (Appendix). To measure CKD severity, we created an indicator for whether most recent estimated glomerular filtration rate (eGFR) in the prior 12 months was less than $30 \mathrm{~mL} / \mathrm{min}$ per $1.73 \mathrm{~m}^{2}$; eGFR was calculated using EHR creatinine lab measures and the CKD-EPI formula [17], excluding the BA/AA race component following recent recommendations [18]. The Charlson score [19], excluding asthma, atherosclerotic cardiovascular disease, CKD, COPD, DM, and HF, was used to summarize additional medical comorbidity burden. Angiotensin-converting enzyme inhibitor (ACEI) and angiotensin II receptor blocker (ARB), oral steroid, and statin use at cohort entry were determined through pharmacy fill data. Neighborhood characteristics were defined using last known address as of March 1, 2020, and information from the 2016 and 2018 US American Community Survey conducted by the US Census Bureau. Neighborhood deprivation index (NDI) [20] was calculated at the census tract using the 2016 American Community Survey and categorized in quartiles. Neighborhood information on the proportion of housing units with more than 1 person per room was calculated at the census tract using the 2018 American Community Survey. See Supplementary Material for detailed variable definitions, including lists of ICD-10 codes used.

\section{Statistical Analyses}

We described the full cohort, those who had a COVID19 infection, and those who had a COVID-19 PCR/NAA test (positive or negative), overall and by racial and ethnic groups. We estimated the relative risk (RR) of severe
COVID-19 across racial and ethnic groups in the full cohort with 5 models, which progressively adjusted for more covariates to understand how each potential confounder set changed RR estimates. Model 1 adjusted for health system only (essentially a proxy for geographic region) and was designed to estimate the relative risk of COVID-19 infection or severe infection, accounting for geographical differences. Model 2 added age modeled with a linear spline (knots at 35, $45,55,65$, and 75 years) and sex; this next adjustment set was selected because the age distribution is quite different across racial and ethnic groups in the US [21] and because age is a well-established risk factor for more severe COVID19 disease. Model 3 added BMI and smoking status; these covariates were considered next as both BMI and smoking are known risk factors for COVID-19 infection and progression to more severe symptoms and their distribution differs between racial/ethnic subgroups. Model 4 added comorbid conditions and use of specific medications (see above) to evaluate if differences in chronic disease burden across racial and ethnic groups explained remaining disparities. Model 5 added NDI and percent of housing units in census tract with more than 1 person per room; these covariates were added to the adjustment set in the last model to evaluate whether after adjusting for all other factors socioeconomic characteristics and household density [21] further explained any differences observed. See Supplementary Material (Table M2) for a summary of the covariate set used in each model.

We fit the same 5 models to estimate RRs for severe COVID-19 among individuals who had a COVID-19 infection, to see if disparities remained. If higher rates of severe COVID-19 among racial and ethnic minority groups, compared to non-HI/LA White individuals, were no longer evident when analyses were restricted to individuals with any COVID-19 infection, this would indicate that disparities were driven by higher infection rates. We fit the same 5 models in the full cohort to estimate RRs for any COVID-19 infection to investigate differences in infection rates across racial and ethnic groups. Lastly, we calculated rates of positive PCR/NAA tests among those people tested, overall and by racial and ethnic groups. If asymptomatic individuals or individuals with mild symptoms from racial or ethnic minorities groups were tested more often compared to non-HI/ LA White individuals, RRs of severe COVID-19 would be biased downwards in the sample of individuals with COVID19 infection and positivity rates would also likely be lower. In all models limited to individuals who had been tested for, or diagnosed with, COVID-19, we additionally adjusted for month of infection or COVID-19 test, because availability of testing varied over our follow-up period.

We modeled all binary outcomes using modified Poisson regression [22] and used inverse probability weights to address missing data [23]. Missing follow-up occurred when individuals disenrolled from their health system before 
September 30, 2020; BMI and NDI also had missing values. Therefore, up to 3 analytic weights were multiplied together to account for missing data; see Supplementary Material (Table M2) for covariates included in missing data models. When the sample was limited to individuals with a PCR/ NAA test, not enough people were missing NDI data to estimate weights; thus, missing NDI weights were not used in this sample. We calculated standard errors using the robust sandwich estimator to account for the mis-specified variance when using a Poisson model for a binary outcome and the analytic missing data weights $[22,23]$. In addition to estimating RRs and 95\% CIs, we marginalized over covariates to estimate absolute risks of outcomes in each racial and ethnic group. To assess impact of shorter prior enrollment times leading to potential covariate measurement error, we conducted sensitivity analyses including only individuals continuously enrolled in their health system since March 1, 2019 (or earlier). Descriptive tables were constructed using $\mathrm{R}$ version 3.6.3 (Vienna, Austria); all other analyses were performed using Stata version 15.1 (College Station, TX).

\section{Role of the Funding Sources}

The funders had no role in selecting health systems, study design, data collection, data analysis, data interpretation, or writing up of the results.

\section{Results}

Our cohort included 1,053,118, individuals (53\% female): Of these, 31.0\% were from KP Colorado, 39.5\% KP Northwest, and 29.5\% KP Washington. The 344 individuals with unknown or non-binary gender were excluded from all analyses; there were no observed COVID-19 infections in this population. The racial and ethnic make-up of our analytic cohort $(N=1,052,774)$ is shown in Table 1 . While the population was $68.7 \%$ White, substantial numbers of individuals from racial and ethnic minority groups were included (e.g., 68,887 Asian American, 41,243 BA/AA, and 93,580 HI/LA individuals). The Supplementary Material (Tables R5 and R6) contains detailed information about individuals who identified as HI/LA and individuals who were classified as multiracial. The full cohort, overall and by racial and ethnic groups, is described in Table 1. Differences were seen across racial and ethnic groups in demographics (e.g., White individuals were older; mean age 53.2 versus $43.8-51.4$ years for all other racial and ethnic groups) and medical conditions (e.g., more BA/AA, IA/AN, and Native Hawaiian/Pacific Islander [NH/PI] individuals had DM; BA/AA: $19.8 \%$, IA/ AN: $19.2 \%$, and NH/PI: $20.8 \%$ compared to $13.1-17.6 \%$ for other groups).
A total of 961,917 (91.4\%) individuals remained enrolled in their health system through the entire follow-up period, and 7,399 people had a COVID-19 infection (see Supplementary Material for description of individuals with COVID-19 PCR/NAA test and a COVID-19 infection). Among the individuals with COVID-19, 1,205 (16.3\%) were hospitalized and 442 people had severe COVID-19 (invasive mechanical ventilation or death). Details of COVID-19 outcomes across racial and ethnic groups are in Supplementary Material (Tables R6 and R7). Striking differences were seen in RRs of severe COVID-19 across racial and ethnic groups; these patterns persisted even after adjusting for demographics, medical comorbidities, and neighborhood information (Table 2). After adjusting for all covariates, Asian American, BA/AA, and HI/LA individuals were twice as likely as Whites to experience severe COVID-19 (Asian American RR: 2.09 [95\% CI:1.36, 3.21]; BA/AA: 2.02 [1.39, 2.93]; HI/ LA: 2.09 [1.57, 2.78]). Examining the impact of adjusting for nested confounder sets, RR estimates changed most after adjusting for age and sex, indicating increased risk compared to White individuals. Further adjusting for BMI, smoking, medical comorbidities, and medication use had less impact on RRs and adjusting for neighborhood characteristics had little impact on RRs.

Restricting to individuals with confirmed COVID-19 (Table 2, bottom half) resulted in RRs for severe COVID-19 closer to 1 . After adjusting for all measured covariates, BA/ AA people had an estimated RR of 0.95 (95\% CI 0.66, 1.37) and for HI/LA individuals the RR was $0.97(0.72,1.31)$. The RR for severe COVID-19 remained elevated for Asian American individuals in this COVID-19-positive cohort (1.82 [1.23, 2.68]).

Risk of COVID-19 infection was increased among Asian American, BA/AA, HI/LA, and NH/PI individuals compared to non-HI/LA White individuals after adjusting for all measured covariates; estimated RRs compared to non-HI/LA White individuals were $1.20(1.08,1.33)$, 1.89 (1.72, 2.09), 2.67 (2.50, 2.84), and 2.49 (2.04, 3.03), respectively (Table 3 ). Table 4 presents estimated absolute rates of severe COVID-19 across racial and ethnic groups and Table 5 reports estimated absolute rates of COVID-19 infection. Among people tested for COVID-19, accounting for all measured covariates, the positivity rate for Asian American individuals was 574 per 10,000 individuals (95\% CI: 519, 629), BA/AA: 686 (627, 745), HI/LA: 926 (880, 972), and NH/PI: 884 (723, 1046), which were all higher than for IA/AN $(376[264,487])$ and non-HI/LA White (394 $[381,407])$ individuals. Adjusting for covariates did not have large impacts on absolute risk estimates of experiencing a COVID-19 infection or of testing positive for COVID-19.

Sensitivity analyses requiring continuous enrollment for 12 months prior to cohort entry produced similar results (Appendix Tables S1-S4). 
Table 1 Characteristics of study population, overall and by race and ethnicity (column percentages)

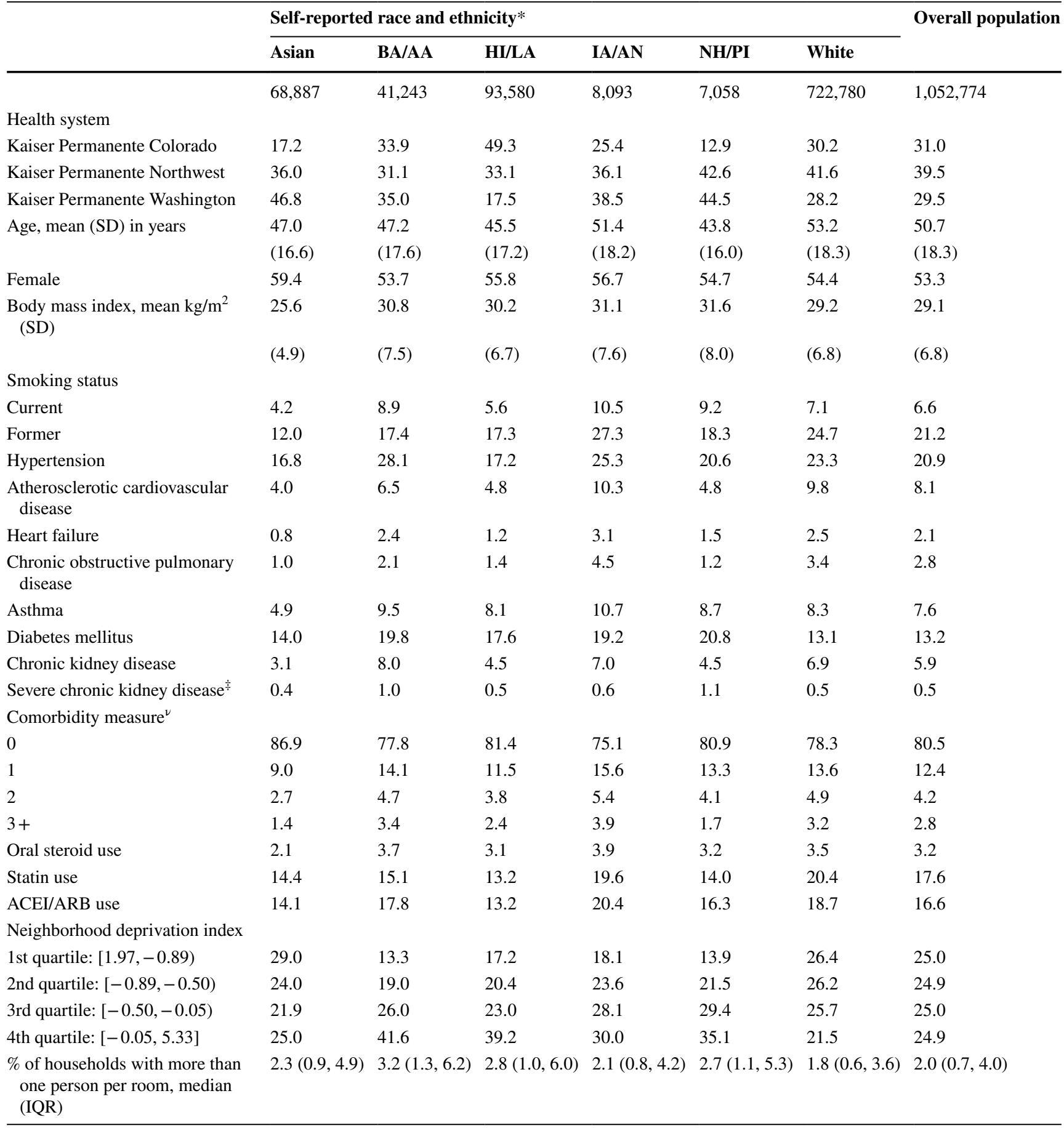

*Individuals self-identified as Hispanic or Latino/Latina (HI/LA) grouped as HI/LA regardless of race selected; all racial groups exclude individuals who identified as HI/LA. BA/AA, Black or African American; IA/AN, Indigenous American/Alaskan Native; NH/PI, Native Hawaiian/ Pacific Islander. Characteristics of individuals who, in our analysis, were classified as multiracial, selected race not listed, and race unknown are in Appendix Table R1

${ }^{\ddagger}$ Severe chronic kidney disease defined as last estimated glomerular filtration rate (eGFR) measured in prior year less than $30 \mathrm{~mL} / \mathrm{min}$ per 1.73 $\mathrm{m}^{2}$ e GFR calculated from creatinine labs using the Chronic Kidney Disease-Epidemiology Collaboration (CKD-EPI) formula excluding the BA/ AA race component of the formula

${ }^{\nu}$ Charlson score excluding asthma, atherosclerotic cardiovascular disease, chronic kidney disease, chronic obstructive pulmonary disease, diabetes mellitus, heart failure

$A C E I$, angiotensin-converting enzyme inhibitor; $A R B$, angiotensin II receptor blockers; $S D$, standard deviation; $I Q R$, interquartile range 
Table 2 Estimated relative risk (95\% confidence intervals) for severe COVID-19

\begin{tabular}{|c|c|c|c|c|c|}
\hline Self-report race/ethnicity & Model 1 & Model 2 & Model 3 & Model 4 & Model 5 \\
\hline \multicolumn{6}{|c|}{ Relative risk of severe COVID-19 in the entire population } \\
\hline Asian American & $1.14(0.77,1.70)$ & $1.96(1.32,2.92)$ & $2.29(1.49,3.51)$ & $2.15(1.40,3.29)$ & $2.09(1.36,3.21)$ \\
\hline Black or African American & $2.06(1.45,2.93)$ & $3.09(2.18,4.38)$ & $2.88(2.00,4.15)$ & $2.31(1.60,3.33)$ & $2.02(1.39,2.93)$ \\
\hline Hispanic or Latino/Latina* & $1.58(1.21,2.06)$ & $2.75(2.11,3.59)$ & $2.71(2.06,3.58)$ & $2.39(1.81,3.17)$ & $2.09(1.57,2.78)$ \\
\hline Indigenous American/Alaskan Native & $1.33(0.50,3.58)$ & $1.65(0.61,4.41)$ & $1.69(0.63,4.55)$ & $1.47(0.55,3.97)$ & $1.39(0.51,3.76)$ \\
\hline Native Hawaiian/Pacific Islander & $0.91(0.23,3.65)$ & $1.96(0.49,7.88)$ & $1.98(0.49,7.99)$ & $1.60(0.40,6.44)$ & $1.52(0.38,6.12)$ \\
\hline White $\dagger$ & REF & REF & REF & REF & REF \\
\hline Multiracial & $0.89(0.13,6.34)$ & $1.41(0.20,9.99)$ & $1.52(0.21,10.83)$ & $1.10(0.15,8.01)$ & $1.02(0.14,7.53)$ \\
\hline Selected race not listed & $1.06(0.56,2.00)$ & $1.57(0.83,2.95)$ & $1.38(0.68,2.80)$ & $1.30(0.64,2.65)$ & $1.26(0.62,2.58)$ \\
\hline Unknown race & $0.50(0.30,0.83)$ & $1.25(0.75,2.09)$ & $1.60(0.75,3.43)$ & $1.76(0.82,3.77)$ & $1.73(0.81,3.71)$ \\
\hline \multicolumn{6}{|c|}{ Relative risk of severe COVID-19 among individuals with confirmed COVID-19" } \\
\hline Asian American & $0.78(0.53,1.14)$ & $1.60(1.13,2.26)$ & $1.88(1.27,2.76)$ & $1.83(1.25,2.69)$ & $1.82(1.23,2.68)$ \\
\hline Black or African American & $0.72(0.52,0.99)$ & $1.16(0.84,1.60)$ & $1.10(0.77,1.58)$ & $1.00(0.70,1.43)$ & $0.95(0.66,1.37)$ \\
\hline Hispanic or Latino/Latina* & $0.53(0.41,0.69)$ & $1.16(0.90,1.50)$ & $1.10(0.83,1.45)$ & $1.01(0.76,1.35)$ & $0.97(0.72,1.31)$ \\
\hline Indigenous American/Alaskan Native & $1.24(0.47,3.24)$ & $2.69(1.02,7.12)$ & $2.69(0.99,7.36)$ & $2.59(0.98,6.83)$ & $2.64(0.99,7.03)$ \\
\hline Native Hawaiian/Pacific Islander & $0.31(0.08,1.24)$ & $0.77(0.19,3.06)$ & $0.93(0.23,3.67)$ & $0.76(0.18,3.22)$ & $0.76(0.18,3.11)$ \\
\hline White $†$ & REF & REF & $\mathrm{REF}$ & REF & REF \\
\hline Multiracial & $0.76(0.10,5.52)$ & $1.15(0.16,8.45)$ & $1.16(0.15,8.88)$ & $0.76(0.08,7.01)$ & $0.67(0.07,6.78)$ \\
\hline Selected race not listed & $0.79(0.43,1.45)$ & $1.16(0.67,1.99)$ & $1.14(0.61,2.13)$ & $1.05(0.57,1.97)$ & $1.06(0.57,1.98)$ \\
\hline Unknown race & $0.61(0.37,1.00)$ & $1.25(0.79,1.98)$ & $1.29(0.68,2.45)$ & $1.24(0.63,2.45)$ & $1.28(0.66,2.47)$ \\
\hline
\end{tabular}

*Individuals self-identified as Hispanic or Latino/Latina (HI/LA) grouped as HI/LA regardless of race selected; all racial groups exclude individuals who identified as Hispanic or Latino/Latina

${ }^{\dagger}$ Reference group non-HI/LA White

Model 1: Adjusted for health system (Kaiser Permanente Colorado, Northwest, or Washington)

Model 2: Adjusted for variables in Model 1, age (linear spline with knots at 35, 45, 55, 65, 75), and sex (male, female)

Model 3: Adjusted for variables in Model 2, health system, age, sex, body mass index $\left(<25,25-30,30+\mathrm{kg} / \mathrm{m}^{2}\right)$, and smoking (current, past, never)

Model 4: Adjusted for variables in Model 3, hypertension, asthma, atherosclerotic disease, chronic kidney disease (CKD), severe CKD, chronic obstructive pulmonary disease (COPD), diabetes mellitus (DM), heart failure (HF), modified Charlson score (Charlson score excluding asthma, atherosclerotic disease, CKD, COPD, DM, HF; categorical as 0,1,2,3+), oral steroid use, angiotensin-converting enzyme inhibitor/angiotensin II receptor blocker use, statin use

Model 5: Adjusted for variables in Model 4, quartile of neighborhood deprivation index, percent in census block with housing units with more than 1 person per room

${ }^{*}$ Models restricted to individuals with COVID-19 adjusted for month of infection in addition to covariates listed

\section{Discussion}

In this large cohort from three US health systems, large differences in risk of severe COVID-19 by self-reported race/ ethnicity were no longer seen in analyses limited to people with documented COVID-19 infection. This finding suggests that disparities in severe COVID-19 in BA/AA and HI/LA individuals were explained by increased rates of COVID19 infection. Increased risk of severe COVID-19 for Asian American individuals was partially, but not fully, explained by increased COVID-19 infection rates. Among individuals who experienced COVID-19, risk of severe COVID-19 outcome was still increased by nearly $80 \%$ in Asian American individuals compared to that in non-HI/LA White individuals. Less representation of people identifying as IA/AN,
$\mathrm{NH} / \mathrm{PI}$, or multiracial in this cohort led to wide CIs. After adjusting for age and gender, the RR of severe COVID-19 in most racial and ethnic groups noticeably increased relative to non-HI/LA White individuals; this is likely because in our cohort, as in the general US population, the age distribution was shifted toward younger ages in Asian American, BA/AA, and HI/LA individuals compared to non-Hispanic White individuals, and younger individuals are less likely to experience severe COVID-19 infection. The proportion of individuals who tested positive for COVID-19 was higher among Asian American, BA/AA, HI/LA, and NH/ PI individuals compared to IA/NA and non-HI\LA White individuals. If "over-testing" (i.e., testing a larger proportion of asymptomatic people or people with mild symptoms) was prominent, we would expect the proportion of individuals 
Table 3 Estimated relative risk (95\% confidence intervals) for any COVID-19 infection

\begin{tabular}{llllll}
\hline Self-report race/ethnicity* & Model 1 & Model 2 & Model 3 & Model 4 & Model 5 \\
\hline Asian American & $1.25(1.13,1.38)$ & $1.18(1.07,1.29)$ & $1.27(1.15,1.42)$ & $1.24(1.11,1.38)$ & $1.20(1.08,1.33)$ \\
Black or African American & $2.47(2.26,2.70)$ & $2.34(2.14,2.56)$ & $2.24(2.03,2.47)$ & $2.11(1.91,2.32)$ & $1.89(1.72,2.09)$ \\
Hispanic or Latino/Latina & $3.43(3.24,3.64)$ & $3.21(3.03,3.40)$ & $3.07(2.89,3.27)$ & $2.98(2.80,3.17)$ & $2.67(2.50,2.84)$ \\
Indigenous American/Alaskan Native & $1.05(0.78,1.41)$ & $1.04(0.78,1.40)$ & $1.06(0.78,1.44)$ & $1.02(0.75,1.39)$ & $0.99(0.73,1.35)$ \\
Native Hawaiian/Pacific Islander & $3.49(2.94,4.15)$ & $3.25(2.73,3.86)$ & $2.82(2.32,3.44)$ & $2.66(2.18,3.24)$ & $2.49(2.04,3.03)$ \\
White† & REF & REF & REF & REF & REF \\
Multiracial & $1.44(1.02,2.02)$ & $1.38(0.98,1.94)$ & $1.32(0.91,1.90)$ & $1.23(0.85,1.78)$ & $1.18(0.82,1.71)$ \\
Selected race not listed & $1.52(1.29,1.80)$ & $1.46(1.24,1.72)$ & $1.53(1.26,1.85)$ & $1.52(1.25,1.84)$ & $1.47(1.21,1.78)$ \\
Unknown race & $0.91(0.83,1.01)$ & $0.84(0.76,0.93)$ & $1.30(1.09,1.55)$ & $1.31(1.10,1.57)$ & $1.26(1.06,1.51)$ \\
\hline
\end{tabular}

*Individuals self-identified as Hispanic or Latino/Latina grouped as such regardless of race selected; all racial groups exclude individuals who identified as Hispanic or Latino/Latina

${ }^{\dagger}$ Reference group non-HI/LA White

Model 1: Adjusted for health system (Kaiser Permanente Colorado, Northwest, or Washington)

Model 2: Adjusted for variables in Model 1, age (linear spline with knots at 35, 45, 55, 65, 75), sex (male, female)

Model 3: Adjusted for variables in Model 2, body mass index $\left(<25,25-30,30+\mathrm{kg} / \mathrm{m}^{2}\right)$, smoking (current, past, never)

Model 4: Adjusted for variables in Model 3, hypertension, asthma, atherosclerotic disease, chronic kidney disease (CKD), severe CKD, chronic obstructive pulmonary disease (COPD), diabetes mellitus (DM), heart failure (HF), modified Charlson score (Charlson score excluding asthma, atherosclerotic disease, CKD, COPD, DM, HF; categorical as $0,1,2,3+$ ), oral steroid use, angiotensin-converting enzyme inhibitor/angiotensin II receptor blocker use, statin use

Model 5: Adjusted for variables in Model 4, quartile of neighborhood deprivation index, percent in census block with housing units with more than one person per room

Table 4 Estimated absolute rates of severe COVID-19 outcome in each racial and ethnic group, marginalizing out over potential confounders. Reported as number out of 10,000 (95\% confidence interval) people

\begin{tabular}{llllll}
\hline Self-report race/ethnicity $\dagger$ & Model 1 & Model 2 & Model 3 & Model 4 & Model 5 \\
\hline Asian American & $4.8(3.0,6.6)$ & $7.0(4.3,9.7)$ & $8.1(4.8,11.4)$ & $7.7(4.6,10.9)$ & $7.7(4.5,10.8)$ \\
Black or African American & $8.5(5.7,11.4)$ & $11.0(7.4,14.7)$ & $10.1(6.6,13.7)$ & $8.3(5.4,11.2)$ & $7.4(4.8,10.0)$ \\
Hispanic or Latino/Latina & $6.6(5.0,8.1)$ & $9.8(7.5,12.2)$ & $9.6(7.2,11.9)$ & $8.6(6.5,10.8)$ & $7.7(5.7,9.6)$ \\
Indigenous American/Alaskan Native & $5.5(0.1,11.0)$ & $5.9(0.1,11.6)$ & $6.0(0.1,11.8)$ & $5.3(0.1,10.5)$ & $5.1(0.1,10.1)$ \\
Native Hawaiian/Pacific Islander & $3.8(0,9.0)$ & $7.0(0,16.7)$ & $7.0(0,16.7)$ & $5.8(0,13.8)$ & $5.6(0,13.3)$ \\
White & $4.2(3.7,4.6)$ & $3.6(3.2,4.0)$ & $3.5(3.1,4.0)$ & $3.6(3.2,4.0)$ & $3.7(3.2,4.1)$ \\
Multiracial & $3.7(0,11.0)$ & $5.0(0,14.9)$ & $5.4(0,15.9)$ & $4.0(0,11.8)$ & $3.7(0,11.2)$ \\
Selected race not listed & $4.4(1.7,7.1)$ & $5.6(2.1,9.1)$ & $4.9(1.5,8.2)$ & $4.7(1.4,8.0)$ & $4.6(1.4,7.9)$ \\
Unknown race & $2.1(1.1,3.1)$ & $4.5(2.3,6.7)$ & $5.6(1.4,9.9)$ & $6.3(1.6,11.1)$ & $6.3(1.6,11.1)$ \\
\hline
\end{tabular}

Estimated absolute risks calculated over the entire follow-up period: March 1, 2020, through September 30, 2020

${ }^{\dagger}$ Individuals self-identified as Hispanic or Latino/Latina grouped as such regardless of race selected; all racial groups exclude individuals who identified as Hispanic or Latino/Latina

Model 1: Marginalized such that racial and ethnic groups have same distribution of health system (Kaiser Permanente Colorado, Northwest, or Washington)

Model 2 Marginalized such that racial and ethnic groups have same distribution of variables in Model 1, age (linear spline with knots at 35, 45, $55,65,75)$, and sex (male, female)

Model 3: Marginalized such that racial and ethnic groups have same distribution of variables in Model 2, body mass index $(<25,25-30,30+\mathrm{kg} /$ $\mathrm{m}^{2}$ ), and smoking (current, past, never)

Model 4: Marginalized such that racial and ethnic groups have same distribution of variables in Model 3, hypertension, asthma, atherosclerotic disease, chronic kidney disease (CKD), severe CKD, chronic obstructive pulmonary disease (COPD), diabetes mellitus (DM), heart failure (HF), modified Charlson score (Charlson score excluding asthma, atherosclerotic disease CKD, COPD, DM, HF; categorical as 0, 1, 2, 3+), oral steroid use, and angiotensin-converting enzyme inhibitor/angiotensin II receptor blocker use, statin use

Model 5: Marginalized such that racial and ethnic groups have same distribution of variables in Model quartile of neighborhood deprivation index and percent in census block with housing units with more than 1 person per room 
Table 5 Estimated absolute rates of positive COVID-19 PCR/NAA tests in each racial and ethnic group, marginalizing out over potential confounders. Reported as number out of 10,000 (95\% confidence interval) tests performed

\begin{tabular}{llllll}
\hline Self-report race/ethnicity $\dagger$ & Model 1 & Model 2 & Model 3 & Model 4 & Model 5 \\
\hline Asian American & $606(553,660)$ & $580(529,631)$ & $589(532,645)$ & $579(524,635)$ & $574(519,629)$ \\
Black or African American & $803(739,866)$ & $780(719,842)$ & $751(687,815)$ & $745(682,808)$ & $686(627,745)$ \\
Hispanic or Latino/Latina & $1133(1082,1184)$ & $1083(1034,1132)$ & $1025(976,1075)$ & $1011(952,1050)$ & $926(880,972)$ \\
Indigenous American/Alaskan Native & $355(252,457)$ & $362(258,466)$ & $377(265,490)$ & $377(265,490)$ & $376(264,487)$ \\
Native Hawaiian/Pacific Islander & $1123(939,1306)$ & $1053(881,1225)$ & $938(765,1112)$ & $928(757,1099)$ & $884(723,1046)$ \\
White & $377(366,389)$ & $385(373,397)$ & $384(371,396)$ & $386(373,398)$ & $394(381,407)$ \\
Multiracial & $484(321,647)$ & $485(321,650)$ & $474(300,648)$ & $477(302,652)$ & $469(298,640)$ \\
Selected race not listed & $642(542,742)$ & $625(528,723)$ & $616(510,722)$ & $611(506,716)$ & $613(508,719)$ \\
Unknown race & $692(627,758)$ & $621(561,680)$ & $601(508,693)$ & $593(502,685)$ & $583(493,674)$ \\
\hline
\end{tabular}

Estimated absolute risks calculated over the entire follow-up period: March 1, 2020, through September 30, 2020

${ }^{\dagger}$ Individuals self-identified as Hispanic or Latino/Latina grouped as such regardless of race selected; all racial groups exclude individuals who identified as Hispanic or Latino/Latina

Model 1: Marginalized such that racial and ethnic groups have same distribution of health system (Kaiser Permanente Colorado, Northwest, or Washington) and month of COVID-19 test

Model 2 Marginalized such that racial and ethnic groups have same distribution of variables in Model 1, age (linear spline with knots at 35, 45, $55,65,75$ ), and sex (male, female)

Model 3: Marginalized such that racial and ethnic groups have same distribution of variables in Model 2, body mass index $(<25,25-30,30+\mathrm{kg} /$ $\mathrm{m}^{2}$ ), and smoking (current, past, never)

Model 4: Marginalized such that racial and ethnic groups have same distribution of variables in Model 3, hypertension, asthma, atherosclerotic disease, chronic kidney disease (CKD), severe CKD, chronic obstructive pulmonary disease (COPD), diabetes mellitus (DM), heart failure (HF), modified Charlson score (Charlson score excluding asthma, atherosclerotic disease CKD, COPD, DM, HF; categorical as 0,1,2,3+), oral steroid use, and angiotensin-converting enzyme inhibitor/angiotensin II receptor blocker use, statin use

Model 5: Marginalized such that racial and ethnic groups have same distribution of variables in Model 4, quartile of neighborhood deprivation index and percent in census block with housing units with more than one person per room

$P C R$, polymerase chain reaction; NAA, nucleic acid amplification

who tested positive for COVID-19 among all those tested would be low. In our cohort, positivity rates indicate that "over-testing" did not lead to capture of more of the lesssevere COVID-19 cases in racial and ethnic minority groups.

Our findings align with literature examining disparities in COVID-19 infections and outcomes in the US. Mackey et al. performed a systematic review of research evaluating racial and ethnic disparities in COVID-19 infection and outcomes [24]. They synthesized the results of 37 articles and reported that BA/AA and HI/LA populations had higher COVID-19 infection risk, hospitalization, and COVID-19-related mortality compared to non-HI/LA White populations, but that case-fatality rates, estimated mostly from in-hospital mortality, were similar across these populations. They reported that Asian populations experienced similar outcomes to non-HI/ LA White populations, but the strength of that evidence was low, and there was insufficient evidence to identify disparities in other populations.

Several articles have appeared since the Mackey et al. review. Ogedegbe et al. reported on a retrospective cohort study of 9,722 patients tested for COVID-19 in the New York University Langone Health System [25]. They reported that the odds of having a positive test were higher among BA/AA and HI/LA patients compared to White patients. The odds of hospitalization, among those patients who tested positive for COVID-19, was similar for BA/AA, HI/LA, and White patients, but was slightly higher among Asian American patients (odds ratio: $1.6[1.1,2.3]$ ), similar to our findings. Escobar et al. found that Asian American, BA/AA, and HI/LA individuals (in Kaiser Permanente Northern California) had higher odds of COVID-19 infection compared to non-HI/LA White individuals, but that there were no differences across racial and ethnic groups in mortality among hospitalized patients [26]. A retrospective study conducted in the US Department of Veterans Affairs by Razjouyan et al. found higher testing positivity rates and increased risk of hospitalization due to COVID-19 among BA/AA and HI/LA veterans, but also found that among those hospitalized there were no differences across race and ethnic groups in intensive care utilization or death [27]. Khanna et al. found, in the population of patients served by the University of Maryland family medicine and immediate care, BA/AA patients and HI/LA patients were more likely to experience COVID-19 infection than non-HI/LA White patients [28]. Khanna et al. also found that patients living in neighborhoods with predominantly Black residents and with a higher deprivation index score had a higher risk of COVID-19 infection compared to patients living in less socioeconomically deprived 
areas that were predominantly White [28]. Similar results found by Khanijahani and Tomassoni showed that counties classified as more socioeconomically and racially segregated neighborhoods had higher rates of COVID-19 deaths [29]. In our analyses, accounting for NDI and percent of households with more than one person per room did not impact RR estimates. It is possible that the reason these factors did not change risk estimates is that the individuals in our population were insured and $70 \%$ of the people in our sample were insured through their employer.

Our study has limitations. First, ethnicity was not collected uniformly within or between healthcare systems. In some clinics, HI/LA ethnicity information was collected as a separate question and in other clinics, HI/LA ethnicity was included as a potential race category. Due to this difference, we categorized all individuals who did not identify as HI/ LA ethnicity as not-HI/LA. Second, interim state death certificates with COD information were not available for KP Northwest. Third, our follow-up period ended in September 2020, and thus did not include the period of significantly higher infections in late 2020-early 2021. Fourth, approximately $70 \%$ of the people in our study were insured through employer-sponsored insurance plans (see Table R1); thus, it is possible that our results may not generalize to an uninsured or underinsured population. Fifth, most individuals these health systems serve live in urban or suburban areas, so we were not able to examine urban-rural differences, and our findings may not be generalizable to rural populations. Sixth, we lacked information about individuals' occupation, an important predictor of potential exposure to COVID-19. This is particularly relevant to our study because people of color may be more likely to work as essential workers and thus to face disproportionately higher risks than more privileged individuals who have been able to work remotely.

Our study had notable strengths. This was a populationbased cohort study of insured individuals enrolled in 3 health systems with comprehensive data capture on covariates and outcomes. These data included laboratory test results for most COVID-19 cases, hospitalization (including procedures performed during hospitalization), and mortality information from hospitalization discharge status and interim state mortality records. Our study used diagnosis codes from hospitalizations that were validated to have high accuracy in these health systems. Our study follow-up period included the beginning of the pandemic when testing was limited as well as the summer of 2020 when testing was more widely available. Access to rich data on all individuals in our cohort meant we could control for confounding by demographic and medical conditions to help address the impact restricted testing early in the pandemic may have had on our results.

Our work continues to highlight the importance of interventions to prevent COVID-19 infection, specifically in racial and ethnic minority communities. A continued focus on equity in vaccine distribution, education, and delivery is essential to achieving health equity for both initial COVID vaccination and subsequent booster shots. Early in 2021, COVID-19 vaccines were difficult to obtain in the US, and required a good internet connection and fluency in English and technology. According to the Centers for Disease Control and Prevention, COVID-19 vaccination rates still vary across racial and ethnic groups [30], but there is evidence that the differences in vaccination rates have narrowed over time [31].

Difficulty in access has been a major cause of unequal COVID-19 vaccination rates; literature points to racial and ethnic minority groups being willing to receive vaccines if offered [32, 33]. Recent US surveys on COVID-19 vaccination have shown an increase over time in the proportion of adults who have had the vaccine or who are willing to take the vaccine when offered [34]. While differences remain in the proportion of Americans willing to receive the COVID19 vaccine across racial and ethnic groups, respondents identified concerns about COVID-19 vaccine efficacy and safety as reasons $[34,35]$. Half of BA/AA and $25 \%$ of HI/LA respondents indicated they were not confident the COVID19 vaccines have been adequately tested among people of their own racial or ethnic group [34, 35]. These concerns emphasize the need for researchers, health systems, and funders to ensure representation from all racial and ethnic groups in clinical trials, to make vaccines easily available to all racial and ethnic groups, and to provide accurate information on vaccine safety and efficacy available to all people, including information about the racial and ethnic make-up of trials.

One unexplained finding, which has limited evidence in prior literature [25], is our finding that Asian Americans may be at a higher risk of severe COVID-19 after infection compared to other racial and ethnic groups. A limitation of our study is that individuals from a variety of backgrounds were grouped together as Asian American. The finding that Asian Americans could be at increased risk of disease progression should be explored in future work, including understanding the impact of anti-Asian racism during the COVID-19 pandemic, identifying potential unknown risk factors that may have disproportionally affected Asian individuals in our cohort, and estimating risk among Asian American subgroups.

Our study adds to understanding of US health inequities of the COVID-19 pandemic, showing that Asian, BA/ AA, HP/PI, and HI/LA individuals were at higher risk of severe COVID-19. We demonstrated that, for most racial and ethnic groups, this increased risk was driven by increased COVID-19 infection risk rather than other factors. It is important to note that during the period of this study there were no effective treatments for mild to 
moderate COVID-19 available that could prevent progression to severe disease. Thus, progression of COVID-19 symptoms to invasive mechanical ventilation or death likely represents the natural progression of disease without intervention. If there had been treatments available that could slow or stop progression to severe disease, we might expect to see disparities in receipt of those treatments by race and ethnicity due to bias and discrimination in healthcare access and treatment [12-14]. If that were the case, then after restricting analyses to individuals infected with COVID-19, we might see continued disparities for severe disease given infection, even if no natural variation in likelihood of disease progression existed. It will be important to monitor for such disparities as new treatments for mild to moderate disease become available, for instance the new oral agents developed by Merck (molnupiravir) and Pfizer (PF-07321332/ritonavir) which are currently under consideration by the FDA for Emergency Use Authorization. Vaccine distribution is an effective way to prevent COVID-19 infection and spread, highlighting the importance of equity in vaccine roll-out, both for initial vaccination and for any recommended booster vaccines. Our study underscores that the social impact of racial and ethnic inequality, as opposed to biological differences, influenced COVID-19 severity.

Supplementary Information The online version contains supplementary material available at https://doi.org/10.1007/s40615-021-01205-2.

Author Contribution All authors confirm that they accept responsibility to submit for publication. The following authors had full access to all the data in the study: SMS, RLW, SF, LT, SPF, LAJ, EAB, LBH, MHL, SD

Conceptualization: SMS, RG, MAA, RLW, SF, LT, SPF, LAJ, JSF, EAB, LBH, SD.

Data curation: SMS, RLW, SF, LT, SPF, LAJ, JSF, EAB, LBH, MHL, SD

Formal analysis: SMS.

Funding acquisition: SPF, EAB, SD.

Project administration: LT, SPF, EAB, SD.

Validation: SF, SPF, LAJ, EAB, SD

Writing-original draft: SMS, RG, MAA, SF, LAJ.

Writing - review and editing: SMS, RG, MAA, RLW, SF, LT, SPF, LAJ, JSF, EAB, LBH, MHL, SD.

Funding This project was supported in all or in part by Kaiser Permanente's Garfield Memorial Fund and Kaiser Permanente Washington Health Research internal funds. National Heart, Lung, and Blood Institute grant K01HL139997 provided partial support for Dr. Laura B. Harrington's time on this study.

Kaiser Permanente's Garfield Memorial Fund,Kaiser Permanente Washington Health Research Institute,National Heart,Lung,and Blood Institute,K01HL139997,Laura B Harrington

\section{Declarations}

Ethics Approval Appropriate Institutional Review Boards approved this study.
Conflict of Interest Financial Interests: SMS was a co-Investigator on Kaiser Permanente Washington Health Research Institute projects funded by Syneos Health, who is representing a consortium of pharmaceutical companies carrying out studies mandated by the US Federal Drug Administration regarding the safety of extended-release opioids. SPF has a research contract from Pfizer|BioNTech as a site for clinical trials of their SARS-CoV-2 RNA vaccine, which is unrelated to the work reported in this paper. JSF has consulted for Shionogi Inc. LT and SD have received research funding on an unrelated topic from GlaxoSmithKline and SD has received funding from Jazz Pharmaceuticals for unrelated work. Non-financial Interests: none.

Disclaimer The funders had no role in selecting health systems, study design, data collection, data analysis, data interpretation, or writing up of the results.

\section{References}

1. Khazanchi R, Evans CT, Marcelin JR. Racism not race drives inequity across the COVID-19 continuum. JAMA Netw Open. 2020;3(9):e2019933.

2. Dorn AV, Cooney RE, Sabin ML. COVID-19 exacerbating inequalities in the US. Lancet. 2020;395(10232):1243-4.

3. Owen WF Jr, Carmona R, Pomeroy C. Failing another national stress test on health disparities. JAMA. 2020;323(19):1905-6.

4. Morales L. Navajo Nation sees high rate of COVID-19 and contact tracing is a challenge. The Coronavirus Crisis [online news article] 2020 [cited 2021 April 15]; Available from: https://www.npr. org/2020/04/24/842945050/navajo-nation-sees-high-rate-ofcovid19-and-contact-tracing-is-a-challenge. Accessed 15 Apr 2021.

5. Yan BW, Ng F, Chu J, Tsoh J, Nguyen T. Asian Americans facing high COVID-19 case fatality. Health Affairs Blog. 2020.

6. Boulware LE. Race disparities in the COVID-19 pandemicsolutions lie in policy not biology. JAMA Netw Open. 2020;3(8):e2018696.

7. Gee GC, Ford CL. Structural racism and health inequities: old issues, new directions. Du Bois Rev. 2011;8(1):115-32.

8. Ferdinand KC, Nasser SA. African-American COVID-19 mortality: a sentinel event. J Am Coll Cardiol. 2020;75(21):2746-8.

9. Hooper MW, Nápoles AM, Pérez-Stable EJ. COVID-19 and racial/ethnic disparities. JAMA. 2020;323(24):2466-7.

10. Williams DR, Cooper LA. COVID-19 and health equity-a new kind of "herd immunity." JAMA. 2020;323(24):2478-80.

11. Acevedo-Garcia D. Residential segregation and the epidemiology of infectious diseases. Soc Sci Med. 2000;51(8):1143-61.

12. Obermeyer $Z$, et al. Dissecting racial bias in an algorithm used to manage the health of populations. Science. 2019;366(6464):447.

13. Bailey ZD, et al. Structural racism and health inequities in the USA: evidence and interventions. Lancet. 2017;389(10077):1453-63.

14. Institute of Medicine (US) Committee on Understanding and Eliminating Racial and Ethnic Disparities in Health Care. Unequal treatment: confronting racial and ethnic disparities in health care. Smedley BD, Stith AY, Nelson AR, editors. Washington (DC): National Academies Press (US); 2003.

15. Rodriguez-Diaz CE, et al. Risk for COVID-19 infection and death among Latinos in the United States examining heterogeneity in transmission dynamics. Ann Epidemiol. 2020;52:46-53e2.

16. Rader B, Astley CM, Sy KTL, Sewalk K, Hswen Y, Brownstein JS, Kraemer MUG. Geographic access to United States SARS$\mathrm{CoV}-2$ testing sites highlights healthcare disparities and may bias transmission estimates. J Travel Med. 2020;27(7):taaa076. https:// doi.org/10.1093/jtm/taaa076. 
17. Levey AS, et al. A new equation to estimate glomerular filtration rate. Ann Intern Med. 2009;150(9):604-12.

18. Ahmed S, et al. Examining the potential impact of race multiplier utilization in estimated glomerular filtration rate calculation on African-American care outcomes. J Gen Intern Med. 2021;36(2):464-71.

19. Charlson ME, et al. A new method of classifying prognostic comorbidity in longitudinal studies: development and validation. J Chronic Dis. 1987;40(5):373-83.

20. Diez Roux AV, et al. Neighbourhood environments and mortality in an elderly cohort: results from the cardiovascular health study. J Epidemiol Community Health. 2004;58(11):917-23.

21. Schaeffer K, The most common age among Whites in U.S. is 58 - more than double that of racial and ethnic minorities. 2019 July 30, 2019 [cited 2021 November 16]; Available from: https://www. pewresearch.org/fact-tank/2019/07/30/most-common-age-amongus-racial-ethnic-groups/. Accessed 16 Nov 2021.

22. Zou G. A modified Poisson regression approach to prospective studies with binary data. Am J Epidemiol. 2004;159(7):702-6.

23. Robins JM, Rotnitzky A, Zhao LP. Estimation of regression coefficients when some regressors are not always observed. J Am Stat Assoc. 1994;89:447-82.

24. Mackey K, et al. Racial and ethnic disparities in COVID-19-related infections, hospitalizations, and deaths : a systematic review. Ann Intern Med. 2021;174(3):362-73.

25. Ogedegbe G, et al. Assessment of racial/ethnic disparities in hospitalization and mortality in patients with COVID-19 in New York City. JAMA Netw Open. 2020;3(12):e2026881.

26. Escobar GJ, Adams AS, Liu VX, Soltesz L, Chen YI, Parodi SM, et al. Racial disparities in COVID-19 testing and outcomes: retrospective cohort study in an integrated health system. Ann Intern Med. 2021;174(6):786-93. https://doi.org/10.7326/M20-6979.

27. Razjouyan J, Helmer DA, Li A, et al. Differences in COVID19-related testing and healthcare utilization by race and ethnicity in the veterans health administration. J Racial Ethn Health Disparities. 2021;1-8. https://doi.org/10.1007/s40615-021-00982-0.

28. Khanna N, Klyushnenkova EN, Kaysin A. Association of COVID19 with race and socioeconomic factors in family medicine. J Am Board Fam Med. 2021;34(Suppl):S40-7.
29. Khanijahani A, Tomassoni L. Socioeconomic and racial segregation and COVID-19: concentrated disadvantage and black concentration in association with COVID-19 deaths in the USA. J Racial Ethn Health Disparities. 2021;1-9. https://doi.org/10.1007/ s40615-021-00965-1.

30. CDC. Demographic characteristics of people receiving COVID19 vaccinations in the United States. COVID Data Tracker 2021 [cited 2021 April 2015]; Available from: https://covid.cdc.gov/ covid-data-tracker/\#vaccination-demographic. Accessed $15 \mathrm{Apr}$ 2021.

31. Ndugga N, et al. Latest data on COVID-19 vaccinations by race/ ethnicity. 2021 November 17, 2021 [cited 2021 December 2]; Available from: https://www.kff.org/coronavirus-covid-19/issuebrief/latest-data-on-covid-19-vaccinations-by-race-ethnicity/. Accessed 2 Dec 2021.

32. Walker ATSP, Kolasa M, National Center for Emerging and Zoonotic Diseases CDC, National Center for Immunization and Respiratory Diseases CDC. Reduction of racial/ethnic disparities in vaccination coverage, 1995-2011. Morbidity and Mortality Weekly Report (MMWR) 2014 [cited 2021 April 15]; Available from: https://www.cdc.gov/mmwr/preview/mmwrhtml/su6301a3. htm?s_cid=su6301a3_x\#tab1. Accessed 15 Apr 2021.

33. Schwartz KL, et al. Racial similarities in response to standardized offer of influenza vaccination. A MetroNet study J Gen Intern Med. 2006;21(4):346-51.

34. Hamel LSG, Brodie M, KFF COVID-19 vaccine monitor: February 2021. Polling 2021 [cited 2021 April 15]; Available from: https://www.kff.org/coronavirus-covid-19/poll-finding/kff-covid19-vaccine-monitor-february-2021/. Accessed 15 Apr 2021.

35. Leipertz GFK, Dominguez A, Locklear S, Ferronato H, EchoHawk A. Results from a national COVID-19 vaccination survey: strengthening vaccine efforts in Indian country. 2021. p. 1-44. https://www.uihi.org/projects/strengthening-vaccine-effor ts-in-indian-country/.

Publisher's Note Springer Nature remains neutral with regard to jurisdictional claims in published maps and institutional affiliations. 